\title{
Variabilité de la durée de la phase végétative chez le pois (Pisum sativum L.). Application à la sélection de types résistants à l'hiver et à la détermination de la date de semis.
}

Gérard ETÉVÉ \& Maurice DERIEUX

avec la collaboration technique de Pascal BIDARD $\left({ }^{*}\right)$, Dominique LAURENT \& Jean-Jacques STEMPNIAK.

I.N.R.A., Laboratoire de Génétique et d'Amélioration des Plantes, Estrées-Mons, F 80200 Péronne.

(*) UNIP (Union Nationale Interprofessionnelle des Protéagineux), 12, avenue Georges-V, 75008 Paris.

\section{RÉSUMÉ}

Pois d'hiver,

Résistance à l'hiver,

Phase végétative,

Initiation florale,

Date de semis.

\begin{abstract}
La résistance au froid du pois est liée au maintien de la plante à l'état végétatif et la durée de la phase végétative est unc caractéristique variétale que l'on peut exprimer en somme de températures.

Ces résultats ont conduit à la sélection, grâce à des semis échelonnés, de types tardifs bien adaptés mais aussi de types très tardifs, ramifiés, présentant en semis d'hiver un rythme d'apparition des nœuds plus lent que les types classiques.

Ces résultats ont permis également pour une région donnée de déterminer la date de semis en fonction du
\end{abstract} niveau de risque accepté et de la durée de la phase végétative de la variété.

\section{SUMMARY}

\section{Winter pea}

Winter hardiness,

Flower initiation,

Vegetative period,

Sowing time.
Variability of the vegetative period in pea (Pisum sativum L.). Application to breeding for winter hardiness and to the determination of sowing date.

Cold resistance of winter pea is effective only during the vegetative period. The duration of this period is a varietal characteristic and it is possible to relate it to heat unit requirements.

Breeding on this basis, with three sowing dates each year, has given late, well adapted, types but also very late, branching types with a slower rate of node differentiation.

These results also allowed sowing date to be determined for a given area, in relation to the accepted level of risk and the duration of the vegetative period.

\section{INTRODUCTION}

Le pois est l'une des espèces qui pourraient augmenter la production nationale de protéines, mais l'irrégularité du rendement est l'un des freins au développement de cette culture.

Chez de nombreuses plantes de grande culture (blé, orge, colza), l'utilisation de types « hiver » a permis d'améliorer à la fois la production et la régularité du rendement. Ces types « hiver », en particulier chez le blé, possèdent la résistance au froid, mais ils ont aussi besoin de températures basses, de vernalisation, pour accomplir leur cycle de développement : semés au printemps, ils ne montent pas. Chez le pois, les variétés semées à l'automne, appelées par commodité «type hiver", n'ont aucun besoin de vernalisation et fleurissent en semis de printemps. La résistance au froid, quant à elle, existe à des degrés divers chez les pois de printemps et chez les pois dits de «type hiver», mais ce n'est pas le seul facteur en cause pour avoir une bonne
" résistance à l'hiver ". En effet, les pois semés actuellement en automne dans le Nord de la France et possédant une bonne résistance intrinsèque au froid (COUSIN, 1976; PRIEUR \& COUSIN, 1978) sont fréquemment détruits par l'hiver. Le plus souvent, les dégâts ne sont pas provoqués par les périodes les plus froides de l'hiver, mais par les gelées du mois de mars auxquelles échappent mieux les génotypes les plus tardifs.

La sélection de pois de type « hiver » est récente, mais il est possible de tirer parti des travaux assez anciens réalisés sur le blé. Par exemple, CREPIN et al. (1929), DEXTER (1956) ont souligné la nécessité d'une sélection pour la résistance à l'hiver et pas seulement pour la résistance au froid. Dionigi (1970) a signalé l'importance de la phase végétative pour la résistance à l'hiver chez le blé.

L'objectif de notre travail est d'étudier, par analogie avec les travaux faits sur le blé, l'influence de la durée de la phase végétative sur la résistance au froid du pois et d'en tirer les conséquences pour la culture et la sélection de types « hiver". 


\section{TABLEAU 1}

Dates d'initiation des 3 variétés de pois en fonction de la date de semis.

Dates of flower initiation for 3 varieties in relation to sowing date.

\begin{tabular}{|c|c|c|c|c|c|c|c|c|c|c|c|c|}
\hline \multirow{2}{*}{$\begin{array}{l}\text { Variété } \\
\text { Variety }\end{array}$} & \multirow{2}{*}{$\begin{array}{l}\text { Type } \\
\text { Type }\end{array}$} & \multirow{2}{*}{$\begin{array}{c}\text { Précocité (1) } \\
\text { Earliness }\end{array}$} & \multicolumn{10}{|c|}{ Date de semis - Sowing date } \\
\hline & & & $14 / 10$ & $29 / 10$ & $12 / 11$ & $16 / 1$ & $5 / 3$ & $24 / 3$ & $14 / 4$ & $7 / 5$ & $5 / 6$ & $22 / 6$ \\
\hline «Frimas" & Hiver lisse jaune & $13-15$ & $10 / 1\left(^{\dagger}\right)$ & $29 / 1\left(^{\dagger}\right)$ & $10 / 2\left(^{*}\right)$ & $29 / 3$ & $11 / 4$ & $25 / 4$ & $14 / 5$ & $31 / 5$ & $24 / 6$ & $9 / 7$ \\
\hline «Frisson » & Hiver lisse jaune & $14-15$ & $19 / 1\left(^{(}\right)$ & - & $6 / 3$ & $1 / 4$ & $11 / 4$ & $25 / 4$ & $14 / 5$ & $30 / 5$ & $25 / 6$ & $9 / 7$ \\
\hline «Vendevil " & Hiver lisse vert & $16-17$ & $10 / 2\left(^{+}\right)$ & $11 / 3$ & $18 / 3$ & $10 / 4$ & $17 / 4$ & $10 / 5$ & $22 / 5$ & - & $2 / 7$ & $14 / 7$ \\
\hline
\end{tabular}

$\left.{ }^{\dagger}\right)$ semis détruits - sowing destroyed

(*) dégâts très importants - very serious damage

(') nœud d'apparition de la première fleur - node of apparition of first flower

\section{MATÉRIEL ET MÉTHODES}

Trois variétés, de précocité différente, « Frimas », « Frisson" et "Vendevil», ont été semées 10 fois entre le 14 octobre 1980 et le 22 juin 1981 à Estrées-Mons (Somme) (latitude : $53^{\circ} \mathrm{N}$, longitude : $3^{\circ} \mathrm{E}$, altitude : $86 \mathrm{~m}$ ).

\section{Observations}

L'observation pratique de la levée est parfois difficile et imprécise, surtout pendant l'hiver, période durant laquelle la levée peut s'étaler sur plusieurs semaines. D'autre part, l'état de différenciation de l'apex est souvent un indicateur plus fidèle de l'âge physiologique de la plante que son aspect extérieur (JONARD et al., 1952). Pour ces deux raisons, la levée a été définie comme la date à laquelle 50 p. 100 des plantes observées ont différencié 9 nœuds.

Des prélèvements de 5 à 6 plantules ont été faits régulièrement (10-15 jours en hiver, 3-4 jours au printemps et en été) pour observer :

- l'apparition successive des nœuds sur l'apex

- l'apparition des premières ébauches florales (initiation florale).

Ces observations reposent sur les descriptions morphologiques faites par NoUGAREDE \& RONDET (1973) et AITKEN (1978).

La fin de la phase végétative est notée quand la moitié des plantes observées est initiée. En fait, il a parfois été nécessaire de déterminer la date d'initiation par interpolation entre les dates d'observation qui encadrent l'initiation (Delecolle \& Gurnade, 1980).

\section{Données météorologiques}

Les sommes de températures sont calculées à partir des moyennes journalières $0,5 \times$ (température max. $t_{x}+$ température min. $t_{N}$ ) (HALlaIRE, 1950), d'après les relevés de la station de Météorologie Nationale de SaintQuentin (latitude : $53^{\circ} \mathrm{N}$, longitude $3^{\circ} \mathrm{E}$, altitude : $98 \mathrm{~m}$ ).

\section{RÉSULTATS}

\section{A. Importance de l'initiation florale}

Les dates d'initiation observées figurent dans le tableau 1. Sachant que le froid est intervenu dans la $2^{\mathrm{e}}$ décade de février, on constate que toutes les variétés semées le 14 octobre avaient atteint l'initiation à cette date et ont été détruites. Pour les 2 semis suivants, seules les variétés dont l'initiation était faite ont été détruites; la variété la plus tardive, "Vendevil ", ne présentait aucun dégât de froid. L'interaction variété $\times$ date de semis est donc très importante.

L'initiation apparaît donc bien comme un "point de virage » (Dionigi, 1970), le passage de l'état végétatif à l'état reproducteur entraînant une perte de résistance au froid.

\section{B. Durée de la phase végétative}

La phase végétative est la période qui précède l'initiation florale. Elle peut être subdivisée en 2 phases : semis-levée et levée-initiation.

Pour caractériser l'influence du climat, nous avons calculé les sommes de températures sur ces périodes en utilisant les températures «air» et «sol » ainsi que différents seuils. Pour comparer les méthodes de calcul, on a cherché à minimiser le coefficient de variation (C.V.) entre les données obtenues sur l'ensemble des semis (Durand, comm. pers.).

\section{Semis-levée}

Pour chacune des dates de semis, la levée des 3 variétés a lieu en même temps. Le tableau 2 montre que la meilleure méthode de calcul des sommes de températures est celle qui utilise les températures «sol avec un seuil de $1{ }^{\circ} \mathrm{C}$. Cela correspond assez bien aux résultats obtenus par BIER-

\section{TABLEAU 2}

Période semis-levée - Coefficients de variation obtenus sur les sommes de température.

Sowing-emergence period - Coefficients of variation obtained with heat units.

\begin{tabular}{ccc}
\hline \hline $\begin{array}{c}\text { Seuil } \\
\text { Threshold }\end{array}$ & $\begin{array}{c}\text { Température sol } \\
\text { soil temperature }\end{array}$ & $\begin{array}{c}\text { Température air } \\
\text { air temperature }\end{array}$ \\
\cline { 2 - 3 } 0 & 10,6 & 17,9 \\
1 & 4,5 & 17,7 \\
2 & 7,7 & 19,1 \\
3 & 16,2 & 22,3 \\
4 & 25,0 & 27,4 \\
\hline
\end{tabular}


HUIZEN (1973) et ANGUS et al. (1980), qui situent les seuils respectivement à $1,2^{\circ} \mathrm{C}$ et $1,4^{\circ} \mathrm{C}$.

La moyenne est de 133 degrés-jour avec un C.V. de 4,5 p. 100.

\section{Levée-initiation}

Les C.V. obtenus avec les différents seuils de calcul des sommes de températures montrent que la meilleure méthode est celle qui utilise les températures " air " avec un seuil de $3{ }^{\circ} \mathrm{C}$ (tabl. 3). La précision est plus grande avec la variété tardive «Vendevil» qu'avec les variétés précoces «Frimas» et «Frisson ». Il peut s'agir d'une interaction génotype $\times$ milieu, mais plus probablement du fait que la phase levée-initiation étant très courte pour les variétés précoces, une erreur d'un jour dans la notation de la levée d'un semis de printemps peut entraîner une erreur de 20 à 25 p. 100 de la durée de la période. On peut donc espérer améliorer la précision en éliminant la plus grande partie de l'erreur due à la notation de levée, c'est-à-dire en considérant la période semis-initiation. Seule subsiste l'erreur liée au changement de méthode de cumul des températures à partir de la levée.

\section{Semis-initiation}

Les C.V. obtenus (tabl. 3 et 4) montrent que la meilleure méthode est celle qui utilise les températures «sol » supérieures à $1^{\circ} \mathrm{C}$ entre le semis et la levée et les températures « air » supérieures à $3{ }^{\circ} \mathrm{C}$ entre levée et initiation. Les C.V. se situent alors entre 3,9 et 8,3 , ce qui est très satisfaisant. Dans ces conditions les moyennes obtenues sont les suivantes :

«Frimas »: 234 degrés-jour ; écart-type 19,4; C.V. 8,3 p. 100

«Frisson": 242 degrés-jour ; écart-type 17,5; C.V. 7,2 p. 100

«Vendevil »: 308 degrés-jour ; écart-type 12,1; C.V. 3,9 p. 100

L'ensemble des résultats obtenus montre que la résistance au froid est liée au maintien de la plante à l'état végétatif et que la durée de la phase végétative est une caractéristique variétale. Par conséquent, contrairement à l'idée émise par STEPONKUS (1978), qui privilégie la résistance au froid, il est intéressant de sélectionner des génotypes à phase végétative

\section{TABLEAU 3}

Coefficients de variation obtenus sur les sommes de températures pour les périodes semis-initiation et levée-initiation. Coefficients of variation obtained with heat unit requirements from sowing to flower initiation and from emergence to flower initiation.

\begin{tabular}{|c|c|c|c|c|c|c|c|c|c|}
\hline \multirow{3}{*}{$\begin{array}{l}\text { Période } \\
\text { Period }\end{array}$} & \multirow{3}{*}{$\begin{array}{l}\text { Variété } \\
\text { Variety }\end{array}$} & \multicolumn{4}{|c|}{$\begin{array}{l}\text { Température sol } \\
\text { soil temperatures }\end{array}$} & \multicolumn{4}{|c|}{$\begin{array}{l}\text { Température air } \\
\text { air temperatures }\end{array}$} \\
\hline & & \multicolumn{4}{|c|}{ seuil - threshold } & \multicolumn{4}{|c|}{ seuil - threshold } \\
\hline & & 0 & 1 & 2 & 3 & 1 & 2 & 3 & 4 \\
\hline $\begin{array}{l}\text { Semis-initiation } \\
\text { Sowing-initiation }\end{array}$ & $\begin{array}{l}\text { «Frimas» } \\
\text { "Frisson" } \\
\text { «Vendevil» }\end{array}$ & $\begin{array}{l}33,8 \\
40,9 \\
24,8\end{array}$ & $\begin{array}{l}32,1 \\
32,9 \\
17,9\end{array}$ & $\begin{array}{l}29,1 \\
26,1 \\
15,4\end{array}$ & $\begin{array}{l}35,2 \\
27,1 \\
24,7\end{array}$ & $\begin{array}{l}24,3 \\
26,2 \\
15,9\end{array}$ & $\begin{array}{r}20,4 \\
19,0 \\
8,5\end{array}$ & $\begin{array}{r}20,0 \\
14,3 \\
7,6\end{array}$ & $\begin{array}{l}22,6 \\
16,7 \\
15,1\end{array}$ \\
\hline $\begin{array}{l}\text { Levée-initiation } \\
\text { Emergence-initiation }\end{array}$ & $\begin{array}{l}\text { «Frimas" } \\
\text { «Frisson" } \\
\text { «Vendevil » }\end{array}$ & $\begin{array}{l}18,3 \\
20,6 \\
15,2\end{array}$ & $\begin{array}{l}15,0 \\
15,7 \\
10,2\end{array}$ & $\begin{array}{l}16,3 \\
14,8 \\
12,4\end{array}$ & $\begin{array}{l}23,7 \\
20,2 \\
21,4\end{array}$ & $\begin{array}{l}14,6 \\
16,9 \\
11,6\end{array}$ & $\begin{array}{r}13,8 \\
13,8 \\
8,5\end{array}$ & $\begin{array}{r}16,1 \\
13,5 \\
9,6\end{array}$ & $\begin{array}{l}20,6 \\
16,3 \\
14,1\end{array}$ \\
\hline
\end{tabular}

TABLEAU 4

Période semis-initiation : coefficients de variation obtenus en associant températures «sol » et « air » dans le calcul des sommes de températures. Period from sowing to flower initiation: Coefficients of variation obtained with heat units based on association of 'air' and 'soil' temperatures.

Seuil - Threshold

Température sol semis-levée

Soil temperature

Sowing-emergence

\section{Température air} levée-initiation

Air temperature

Emergence-initiation
« Frimas "

« Frisson»

«Vendevil »

$\begin{array}{rrrr}12,6 & 9,3 & 7,5 & 8,3\end{array}$

$\begin{array}{llll}14,9 & 10,9 & 8,4 & 7,8\end{array}$

$\begin{array}{rrrr}11,5 & 6,8 & 4,6 & 6,4\end{array}$

1

2

=._._._._.


longue pour résister à l'hiver et, par ailleurs, de moduler les dates de semis en fonction des génotypes et des régions. Nous allons étudier un exemple de ces 2 types d'application.

\section{APPLICATIONS}

\section{A. Mise au point d'une méthode de sélection pour la résistance à l'hiver}

Les dégâts résultant de l'interaction précocité $\times$ date de semis $\times$ date de gelée, nous avons utilisé, pour réaliser une sélection efficace et régulière, une technique de semis échelonnés des pépinières de sélection.

Dès la $F_{2}$, on sème la descendance d'une plante 3 ou 4 fois à $15 \mathrm{j}$ d'intervalle. A chaque semis, 5 à 6 grains sont semés, de telle sorte que la descendance d'une plante est représentée sur le terrain par une ligne de 3 à 4 poquets d'âge différent. Il est bien sûr nécessaire de répéter assez fréquemment sur le terrain une gamme de témoins pour contrôler l'hétérogénéité du terrain et définir localement l'intensité de sélection.

\section{Résultats}

Une population à 8 parents, associant variétés cultivées classiques et populations locales résistantes à l'hiver, a été constituée par croisement pyramidal à partir de 1976 et observée selon cette méthode. Soixante-huit lignées ont été sélectionnées sur 10000 plantes de départ. Certaines ont une période végétative très longue (fig. 1). Parmi ces lignées se trouvent des types fourragers à fort développement végétatif, des lignées classiques (M 226) et des lignées très tardives (M 130) intéressantes à 3 points de vue :

- La durée de la phase végétative (somme de températures levée-initiation supérieure à 300 degrés-jour) leur confère un avantage pour la résistance à l'hiver. Cette tardiveté retarde la date de la récolte, ce qui risque de poser des problèmes sanitaires (attaques d'oïdium).

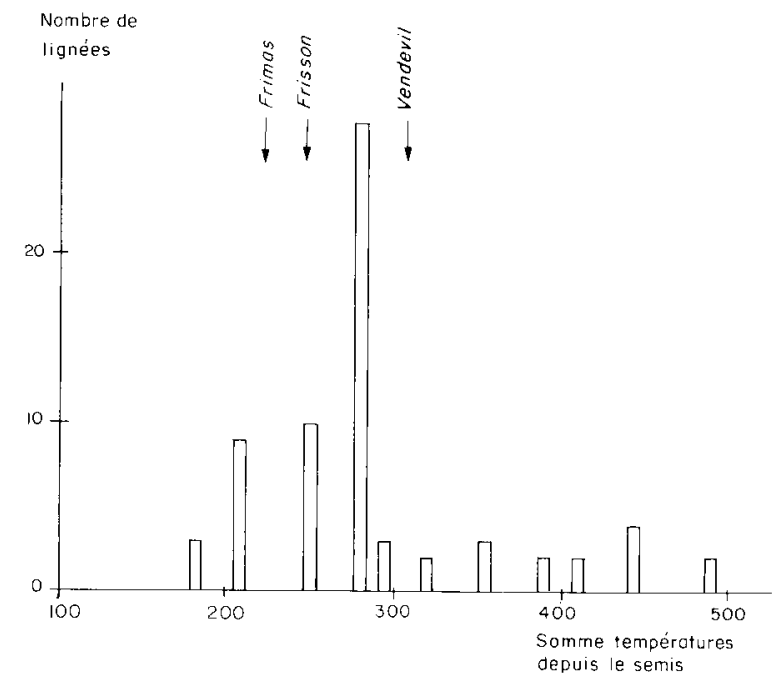

Figure 1

Répartition des 68 lignées sélectionnées pour la résistance à l'hiver en fonction de la durée de la phase végétative.

Distribution of 68 lines selected for winter hardiness in relation to duration of vegetative period.
- La ramification très abondante assure une meilleure stabilité au vent. Ce caractère avait déjà été observé par KHITRINSKII (1960), MARKARIAN \& ANDERSEN (1966), MARKARIAN et al., (1968), ANDERSEN \& MARKARIAN, (1968). Dillman, (1941) avait déjà remarqué chez le lin que les variétés ramifiées résistaient mieux à l'hiver. A la sortie de l'hiver, les plantes de type classique sont déchaussées ; le vent provoque des dégâts mécaniques sur le collet, en particulier des décollements de lépiderme qui sont des voies de pénétration pour les agents pathogènes.

- En semis d'hiver le rythme d'apparition des nœuds (fig. 2, droite 1) est différent de celui des génotypes classiques (droite 2). Par contre, en semis de printemps, le rythme d'apparition des nœuds est le même pour tous les génotypes (droite 3).

Cela remet en cause l'observation classique de précocité basée sur le numéro d'ordre du nœud portant la $1^{\text {re }}$ fleur (COLlins \& Wilson, 1974).



Figure 2

Rythme d'apparition des næuds

$\triangle$ variétés "hiver" classiques "Frimas", "Frisson", "Vendevil»

O Variétés tardives ramifiées: "Winkossa », "M 130 ", "M 64 "

p Variété "printemps": "Finale »

$\triangle O$ semis printemps

$\Delta$ semis hiver

Rate of node differentiation

$\triangle$ Usual winter varieties "Frimas", "Frisson", "Vendevil".

O Late-branching varieties: "Winkossa" "M.30", "M.64".

p Spring variety: "Finale"

$\triangle O$ spring sowing

$\Delta \bullet$ winter sowing

\section{B. Détermination de la date de semis en fonction de la variété et de la région}

La faculté pour un pois (résistant au froid) de passer l'hiver étant liée à son maintien à l'état végétatif, il es̀t intéressant de définir, pour une région donnée, le niveau de risque correspondant à une date de semis, connaissant la durée de la phase végétative d'une variété.

Les données météorologiques de St-Quentin sur les 25 dernières années montrent qu'il n'y a plus de risque de gelée importante $\left\{0,5\left(\mathrm{t}_{\mathrm{X}}+\mathrm{t}_{\mathrm{N}}\right)<0{ }^{\circ} \mathrm{C}\right\}$ après la dernière décade de mars. Nous avons par conséquent calculé les sommes de températures, selon la méthode que nous avons définie, pour chaque année à partir de fin mars jusqu'au mois d'octobre de l'année précédente. 
TABLEAU 5

Nombre d'années où l'initiation aurait eu lieu avant la fin du mois de mars à St-Quentin pour les 25 dernières années. Frequency of flower initiation before end of March for the last 25 years at St-Quentin.

\begin{tabular}{|c|c|c|c|c|c|c|c|c|c|c|}
\hline \multirow{2}{*}{$\begin{array}{l}\text { Variété } \\
\text { Variety }\end{array}$} & \multirow{2}{*}{$\begin{array}{l}\text { Durée de la phase } \\
\text { végétative en } \\
\text { degré jour } \\
\text { Vegetative period - } \\
\text { duration } \\
\text { degree-day }\end{array}$} & \multicolumn{9}{|c|}{$\begin{array}{l}\text { Date de semis } \\
\text { Sowing date }\end{array}$} \\
\hline & & $1 / 1$ & $20 / 12$ & $10 / 12$ & $1 / 12$ & $20 / 11$ & $10 / 11$ & $1 / 11$ & $20 / 10$ & $10 / 10$ \\
\hline «Frimas » & 234 & $13(1)$ & $17(4)$ & $21(6)$ & $23(9)$ & $24(15)$ & $24(19)$ & $25(22)$ & $25(25)$ & $25(25)$ \\
\hline «Vendevil » & 308 & $1(0)$ & $6(1)$ & $12(1)$ & $12(1)$ & $19(2)$ & $19(5)$ & $23(8)$ & $25(17)$ & $25(25)$ \\
\hline «M $226 »$ & 390 & $0(0)$ & $0(0)$ & $1(0)$ & $3(0)$ & $5(1)$ & $7(2)$ & $12(2)$ & $22(4)$ & $25(23)$ \\
\hline «M $130 »$ & 480 & $0(0)$ & $0(0)$ & $0(0)$ & $0(0)$ & $0(0)$ & $1(0)$ & $3(1)$ & $8(2)$ & $22(9)$ \\
\hline
\end{tabular}

( ) nombre d'années où la culture aurait été détruite.

( ) Frequency of crop destruction in the same period.

L'objectif est donc de déterminer la date de semis pour que l'initiation florale n'intervienne pas avant cette date. Les risques d'une initiation florale avant fin mars pour 4 variétés, «Frimas» (précoce), «Vendevil» (assez tardive), «M 226 » (tardive), «M 130» (très tardive, type «Winkossa») sont donnés dans le tableau 5. En fait, il s'agit là d'un risque potentiel, il n'y a de dégâts importants que si une gelée se produit effectivement après initiation (tabl. 5). Concrètement sur ces 25 dernières années, pour un semis de début novembre, «Frimas" aurait été détruit 22 fois, «Vendevil » 8 fois, «M 226 » 2 fois, « M 130 » 1 fois.

Par conséquent, il faut réserver l'implantation des types précoces aux zones froides, où il n'y a pas de risque d'initiation florale avant la fin de l'hiver, et retarder les semis dans les zones à hivers plus « océaniques ».

Cet exemple est basé sur les résultats obtenus en un seul lieu. Il faudrait généraliser la méthode en l'appliquant à d'autres régions. On pourrait ainsi «cartographier » les dates de semis en fonction des variétés et du niveau de risque accepté.

En conclusion, on voit l'intérêt pour développer la culture du pois semé en automne de prendre en compte non seulement la résistance au froid mais aussi la durée de la phase végétative et les caractéristiques climatiques des régions où l'on veut réaliser cette culture. Ceci est particulièrement important pour la sélection de génotypes capables de supporter régulièrement l'hiver.

\section{Reçu le 15 décembre 1981} Accepté le 27 mai 1982.

\section{REMERCIEMENTS}

Nous remercions H. Bannerot, R. Bonhomme, P. Pluchard pour leurs conseils. Ce travail a bénéficié du mémoire de fin d'études de P. Delcourt.

\section{RÉFÉRENCES BIBLIOGRAPHIQUES}

Aitken Y., 1978. Flower initiation in relation to maturity in crop plants. IV. Sowing time and maturity type in pea (Pisum sativum L.) in Australia. Aust. J. agric. Res., 29, 983-1001.

Andersen R. L., Markarian D., 1968. The inheritance of winter hardiness in Pisum. III. Stem branching in autumn growth. Euphytica, 17, 473-478.

Angus J. F., Cunningham R. B., Moncur M. W., Mackenzie D. H., 1980. Phasic development in field crop. I. Thermal response in the seedling phase. Field Crops Res., 3, 365-378.

Bierhuizen J. F., 1973. The effect of temperature on plant growth, development and yield. Actes du colloque d'Uppsala. Réponse des plantes aux facteurs climatiques. UNESCO, ed. 89-98.

Collins W. J., Wilson J. H., 1974. Node of flowering as an index of plant development. Ann. Bot., 38, 175-180.

Cousin R., 1976. Amélioration génétique du pois d'hiver. Ann. Amélior. Plantes, 26, (2), 235-263.

Crépin Ch., Alabouvette L., Meneret G., Chevalier R., 1929. La résistance au froid chez le blé et l'avoine. Ann. Sci. Agron. fr. et étrang., 6, 661-718.

Delecolle R., Gurnade J. C., 1980. Liaisons entre le développement et la morphologie du blé tendre d'hiver. I. Stades de développement de l'apex, apparition des feuilles et croissance de la tige. Ann. Amélior. Plantes, 30 (4), 479-498.

Dexter S. T., 1956. The evaluation of crop plants for winter hardiness. Adv. Agron., 8, 203-237.

Dillman A. C., 1941. Cold tolerance in flax. J. Am. Soc. Agron., 33, 787-799.
Dionigi A., 1970. Résultats de neuf années de recherches du groupe de travail "climat et rendement" sur le « rythme de développement du blé ». Eucarpia Proc. Meet. sections cereals and physiology, Dijon, 135-161.

Durand R., 1967. Action de la température et du rayonnement sur la croissance. Ann. Physiol. vég., 9 (1), 5-27.

Hallaire M., 1950. Les températures moyennes nocturnes, diurnes et nycthémérales exprimées en fonction du minimum et du maximum journaliers de température. C.R. Acad. Sci. Paris, 231, 101104.

Jonard P., Koller J., Vincent A., 1952. Evolution de la tige et de l'épi chez la variété de blé "Vilmorin 27 » au cours de la période de reproduction. Ann. Amélior. Plantes, 1, 1-24.

Khitrinskii V. F., 1960. Guided modification of the heredity of nonwintering pea plants to overwintering pea plants. Agrobiolo giya, 6, 860-865.

Markarian D., Andersen R. L., 1966. The inheritance of winter hardiness in Pisum. Euphytica, 15, 102-110.

Markarian D., Harwood R. R., Rowe PH. R., 1968. The inheritance of winter hardiness in Pisum. II. Description and release of advance generation breeding lines. Euphytica, 17, 110-113.

Nougarede A., Rondet P., 1973. Les. stipules de Pisum sativum L. var. "Nain hâtif d'Annonay » et leurs relations avec la feuille à l'état jeune. C.R. Acad. Sci. Paris, 277, série D, 393-396.

Prieur R., Cousin R., 1978. Contribution à la mise au point d'une technique de sélection pour la résistance au froid des pois d'hiver. Ann. Amélior. Plantes, 28 (2), 157-163.

Steponkus P. L., 1978. Cold hardiness and freezing injury of agronomic crops. Adv. Agron., 30, 51-98. 